Arthroskopie 2021 · 34:351-357

https://doi.org/10.1007/s00142-021-00467-6

Angenommen: 16. April 2021

Online publiziert: 12. Mai 2021

() Der/die Autor(en) 2021

\section{Redaktion}

M. Hirschmann, Basel

J. Agneskirchner, Hannover

Céline S. Moret · Michael T. Hirschmann

Klinik für Orthopädie und Traumatologie, Kantonsspital Baselland, Bruderholz, Schweiz

\title{
Navigation und Robotik in der Knieendoprothetik
}

rurgen durchgeführt werden, signifikante Abweichungen in der postoperativen mechanischen Achse von mehr als $3^{\circ}$ auf [64]. Eine Fehlstellung der unteren Extremität verändert die Kräfteverteilung über das Knie und fördert dadurch die Abnutzung der Prothese und Instabilitäten [6].

\) Eine optimale Beinachse wird
durch die ideale Positionierung
der Prothesenkomponenten
erreicht
Dennoch klagen 20-30\% der Patienten nach einer TEP über Schmerzen und mit der Zeit über Einschränkungen in der Funktion [2, 7, 20, 24, 57]. Innerhalb der ersten 10 Jahre nach der Erstimplantation müssen sich bis zu $10 \%$ der Patienten wegen Versagens der Prothese einer Revision unterziehen [13, 44, 50]. Verantwortlich dafür sind in Deutschland v. a. Lockerungen (23,9\%), Infektionen (14,5\%) und Bandinstabilitäten (8,5\%) [17]. Bei der unikondylären Kniearthroplastik (UKA) sind die Versagensraten höher als bei der TEP und bereits in der Frühphase zu erkennen [17].

Eine stark von der neutralen mechanischen Achse abweichende Beinachse ist eine wichtige Ursache für lockerungsbedingte Revisionen [40]. In Studien wurde aufgezeigt, dass bereits eine Achsenabweichung von $\pm 3^{\circ}$ in der koronaren Ebene das Risiko eines Versagens der Prothese erhöhen kann [37, 40, 44]. Trotz der Verwendung von intra- oder extramedullären Ausrichtungsstäben treten schätzungsweise bei mindestens $10 \%$ der konventionellen TEP (konv-TEP), und zwar auch bei jenen, die von erfahrenen Chi-

\section{Computerassistierte orthopädische Chirurgie}

Es gibt 3 Arten von computerassistierter Chirurgie: Die Navigation unterstützt den Chirurgen bei der präoperativen Planung, der chirurgischen Simulation oder der intraoperativen Führung. Die einzelnen Operationsschritte liegen in der Hand des Chirurgen, weshalb das System passiv genannt wird. Vor der Operation wird eine Computertomographie (CT) und eine dreidimensionale (3-D) Computerplanung des Knies angefertigt [63]. Intraoperativ werden Sensoren an das Femur und an die Tibia fixiert und von einer an einen Computer gekoppelten stereotaktischen Infrarotkamera erkannt (• Abb. 1). Anatomische Orientierungspunkte werden darauf von einem Pointer abgefahren, der von der Kamera erkannt wird, so dass der Computer die Knochensegmente und das Kniegelenk in 3-D im Raum rekonstruieren kann. Die Operation wird dann unter Visualisierung des rekonstruierten Gelenks auf dem Bildschirm durchgeführt. Der Chirurg führt die Knochenschnitte mit der Säge selbst durch, wobei Instrumente verwendet werden, die es dem Chirurgen ermöglichen, jederzeit die korrekte Durchführung der Operation zu überprüfen.

Die Robotik wird in aktive und semiaktive Systeme unterteilt [58]. Beide benötigen eine ausführliche präoperative Planung und intraoperative Navigation.

Beim semiaktiven System bewegt der Chirurg selbst die Säge oder Fräse, die an einem artikulierten Arm befestigt ist, wobei er in seiner Bewegungsfreiheit entsprechend den präoperativ festgelegten Grenzen eingeschränkt wird (• Abb. 2). 


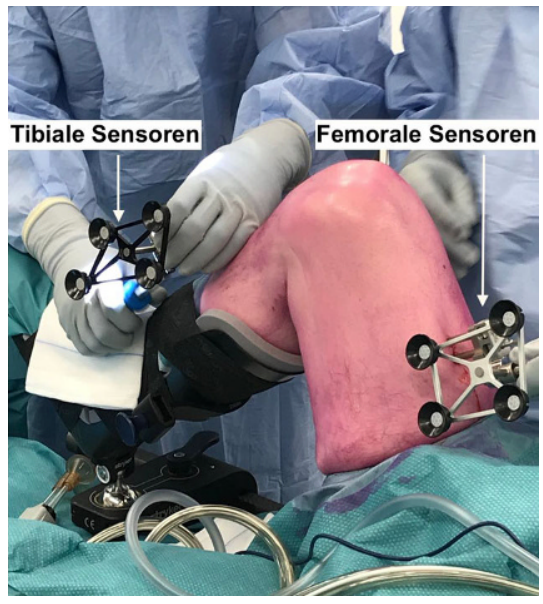

Abb. $1 \Delta$ Die Position der an Femur und Tibia fixierten Sensoren wird durch eine stereotaktische Kamera registriert. Dies ermöglicht die Lokalisation des Femurs und der Tibia im Raum. (Mit freundlicher Genehmigung Dr. Nanne Kort, CortoClinics, Schijndel/NL)

Das System stoppt die Bewegungen des Chirurgen, wenn der vorher festgelegte Weg überschritten wird.

Beim aktiven System führt ein artikulierter Arm alleine unter visueller Kontrolle des Chirurgen die Knochenschnitte selbstständig durch.

\section{Computerassistierte vs. konventionelle Technik}

\section{Alignement und Positionierung der Prothesenkomponenten}

\section{Navigation}

Gemäß mehreren Studien erfolgt bei der nav-TEP eine präzisere Annäherung der postoperativen Beinachse an die neutrale mechanische Achse [42, 43]. Die Mehrheit der Autoren ist sich darin einig, dass die Streubreite um die neutrale, mechanische Achse mit der Navigation kleiner wird [4, 8]. In der Metaanalyse von Bauwens et al. [4] wurde aufgezeigt, dass trotz einer gleichen, gemittelten postoperativen Beinachse in beiden Gruppen dank der Navigation $25 \%$ weniger Knie eine Abweichung der neutralen mechanischen Achse von $3^{\circ}$ bzw. $2^{\circ}$ aufwiesen.

Allgemein wird die Ausrichtung und die Positionierung der Prothesenkomponenten mittels nav-TEP präziser, da Winkelabweichungen vom präoperativ geplanten Ziel und der Anteil der Ausreißer verringert werden. Divergenzen

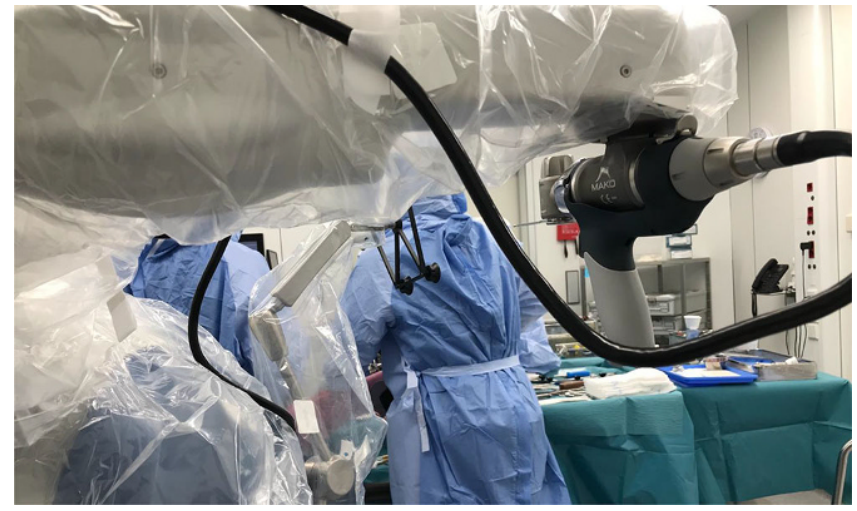

Abb. $2 \Delta$ Semiaktives Robotersystem Mako ${ }^{\circledR}$ (Stryker Corporation, Kalamazoo, Michigan, USA). Links unten befindet sich die Steuerzentrale mit dem artikulierten Arm, der die Fräse oder Säge trägt. Der Chirurg wird somit in seiner Bewegungsfreiheit entsprechend den präoperativ festgelegten Grenzen eingeschränkt. (Mit freundlicher Genehmigung Dr. Nanne Kort, CortoClinics, Schijndel/NL)

ergaben sich v. a. bei den einzelnen Komponenten und Ebenen [43, 65]. Gemäß der Metaanalyse von Mason et al. [42] wurden Abweichungen von $\pm 2^{\circ}$ bzw. $\pm 3^{\circ}$ in allen Ebenen signifikant reduziert, mit Ausnahme der tibialen Neigung (Slope). Andere Autoren stellten hingegen fest, dass der tibiale Slope näher bei den gewünschten $5^{\circ}$ lag [65]. Die Präzision der koronaren und sagittalen Ausrichtung der femoralen Komponente wurde mit der Navigation erhöht, jedoch nicht jene der tibialen [43]. Im Gegensatz dazu berichteten Tandogan et al. [65], dass die Navigation die Genauigkeit der Rotationsausrichtung der tibialen Komponente verbessern kann und dass die Rotationsausrichtung beider Komponenten in beiden Gruppen gleich war.

\section{Robotik}

Marchand et al. [41] führten eine Serie von 330 rob-TEP durch, wobei 129 Knie einen schweren Varus und 7 einen schweren Valgus aufwiesen, alle mit Abweichungen von $>7^{\circ}$. Im Endergebnis konnten dank der Robotik $64 \%$ der schweren Varusknie, alle nichtschweren Varusknie und alle Valgusknie in die neutrale Position korrigiert werden. Diese Feststellung wird auch von anderen Autoren bestätigt $[60,68]$. Zudem gab es in der rob-TEPGruppe keine Ausreißer $\left(> \pm 3^{\circ}\right)$ in der Beinachse [61].

Eine randomisierte kontrollierte Studie von Kim et al. [34] mit 1516 Patienten über 65 Jahre und einer mittleren Verlaufszeit von 13 Jahren konnte nachwei- sen, dass der femorotibiale Winkel, die Positionierung der femoralen und tibialen Komponente in der koronalen und sagittalen Ebene in beiden Gruppen identisch waren.

Lee et al. [38] fanden hingegen in randomisierten kontrollierten Studien keinen signifikanten Unterschied bezüglich Ausreißern der Beinachse sowie der Femur- und Tibiakomponenten in der koronalen Ebene.

Gemäß einigen Studien konnte mit der rob-TEP erfolgversprechend eine verbesserte Spalt- und Weichteilbalancierung erreicht werden $[27,60,61]$.

\section{Klinische Ergebnisse und PROMs}

\section{Navigation}

Obwohl die Ausrichtung der Beinachse und die Positionierung der Prothesenkomponente insgesamt verbessert wurde, scheint sich dies in den Ergebnissen zur Funktion und in der individuellen Wahrnehmung der Patienten (PROMs) nicht widerzuspiegeln.

In einer Gruppe von 60 Patienten, die sich einer bilateralen Knie-TEP unterziehen mussten, wurde ein Knie konventionell und das andere navigiert operiert [21]. Auch nach 8 Jahren wurde keine Verbesserung der klinischen Ergebnisse erzielt. Angewendet wurden der Hospital for Special Surgery Score (HSS), der Western Ontario and McMaster Universities Osteoarthritis Index (WOMAC), der Short form-36 sowie der International Knee Society Score (IKS). Eine Au- 
torengruppe um Panjwani et al. [53] hat in einer Metaanalyse aus (18 qualitativen Studien) mit 3060 Knien die funktionellen Ergebnissen wie der "range of motion “ (ROM), der Knee Society Score (KSS), der WOMAC ermittelt. In den Verlaufskontrollen bis 5 Jahre gab es keinen Unterschied zwischen den beiden Gruppen, zwischen 5 und 8 Jahren konnten sie jedoch eine signifikante Verbesserung des WOMAC und des Function Score des KSS in der nav-TEP-Gruppe identifizieren [51]. Weitere Autoren kamen ebenfalls zum Schluss, dass beide Techniken auch noch nach 10 bis 12 Jahren gleiche Ergebnisse liefern [8, 14, 33, 38, 46, 55].

\section{Robotik}

Yang et al. [68] beobachteten in einer retrospektiven Studie mit 113 Knien bis 10 Jahre postoperativ keinen Unterschied zwischen den beiden Gruppen hinsichtlich WOMAC, ROM, HSS, der Schmerzintensität sowie der Komplikationen. Weitere Studien zeigen das gleiche Resultat [60, 61]; so Kim et al. [34], die in einer randomisierten kontrollierten Studie mit 1516 Patienten unter 65 Jahren nach einer mittleren Verlaufszeit von 13 Jahren ebenfalls keine signifikanten Unterschiede in Bezug auf KSS, WOMAC und ROM feststellten.

\section{Peri- und intraoperative Ergebnisse}

\section{Navigation}

Der Großteil der Studien berichtet über eine signifikant verlängerte Operationszeit, wobei diese mit steigender Erfahrung auf $6-18 \mathrm{~min}$ sinkt $[4,12,14,21$, 46, 55].

In einer retrospektiven Studie mit 878 TEP kam es bei $2,3 \%$ der Knie intraoperativ zu Lockerungen des Referenzstabes, Brüchen des Femurs und Ausfällen des Informatiksystems, die intraoperativ einen Wechsel zur konventionellen Technik erforderten [49]. Das Auftreten von postoperativen thrombembolischen Ereignissen und Protheseninfektionen ist bei beiden Verfahren gleich zu bewerten $[1,46]$. Die Navigation führt anscheinend zu weniger Hämoglobinabfällen [21].

Arthroskopie 2021 · 34:351-357 https://doi.org/10.1007/s00142-021-00467-6

(c) Der/die Autor(en) 2021

\section{S. Moret $\cdot$ M. T. Hirschmann}

\section{Navigation und Robotik in der Knieendoprothetik}

\section{Zusammenfassung}

Hintergrund. Die Langlebigkeit einer Knieprothese hängt von der korrekten Ausrichtung der Prothesenkomponenten und der mechanischen Achse des Beins sowie einer optimalen Weichteilbalancierung ab. Um dieses Ziel präziser und sicherer zu erreichen, wurde die computerassistierte Chirurgie entwickelt. Ziel dieses Artikels ist es, die Navigation und Robotik zu bewerten und die aktuellen Ergebnisse zu diskutieren.

Methode. Umfassende Literaturrecherche in der Datenbank PubMed und dem Suchdienst Google Scholar.

Ergebnisse. Die computerassistierteChirurgie führt zu einer präziseren Ausrichtung der mechanischen Beinachse und der Positionierung der Prothesenkomponenten. Trotzdem sind die klinischen Ergebnisse zur Kniefunktion und die Resultate der patientenbezogenen Fragebögen (PROMs) kontrovers. Sie zeigen, wie für die Überlebensrate, keinen signifikanten Unterschied zur konventionellen Technik. Hohe Anschaffungsund Unterhaltskosten limitieren zudem die Verbreitung dieser Systeme.

Schlussfolgerung. Momentan bietet die bildgestützte Navigation dem Chirurgen bei gewissen Operationsschritten eine wertvolle Unterstützung für ein präziseres und sichereres Arbeiten. Trotzdem sind Langzeitstudien zur Kniefunktion, zu den PROMs, zur Überlebensrate dringend erforderlich. In der Robotik zeigen nur kurzfristige Ergebnisse ähnliche Trends wie bei der Navigation. Die Robotiksysteme müssen und werden sich weiterentwickeln, um den Anforderungen der Nutzer und Patienten zu entsprechen (Kosten, Größe, Programmierung).

\section{Schlüsselwörter}

Kniegelenk · Computerassistierte Chirurgie - Bildgestütztes Navigationssystem . Unikondyläre Prothetik $\cdot$ Kosten

\section{Navigation and robotics in knee arthroplasty}

\section{Abstract}

Background. The longevity of a knee prosthesis depends on the correct alignment of the prosthetic components and the mechanical leg axis as well as an optimal soft tissue balancing. Computer-assisted surgery has been developed to achieve this goal more precisely and safely. The aim of this article is to assess the navigation and robotics and to discuss the current results.

Methods. Comprehensive literature search in the PubMed database and the Google Scholar search service.

Results. Computer-assisted surgery leads to a more precise alignment of both the mechanical leg axis and the positioning of prosthetic components. Nevertheless, the clinical results for knee function and the results of patient reported outcome measures (PROM) are controversial. They show no significant differences to conventional techniques and the same applies for the survival rates. High acquisition and maintenance costs limit the dissemination of these systems.

Conclusion. At present, imaging-assisted navigation provides the surgeon with valuable support for more precise and safe work with respect to specific surgical steps. Nevertheless, long-term studies on knee function, PROMs and survival rate of the prosthesis are urgently needed, especially in robotics, even if the short-term results show trends similar to those for navigation. Robotic systems certainly need further development to meet the requirements of users and patients (costs, size, programming).

\section{Keywords}

Knee joint - Computer-assisted surgery · Image-based navigation system . Unicompartmental knee arthroplasty · Costs

\section{Robotik}

Die Operationszeit ist auch bei der robTEP deutlich verlängert (25 min; [61]). Bezüglich Komplikationen gab es keinen Unterschied zwischen den beiden Gruppen $[20,37,38]$, und in der rob-TEPGruppe war der intra- und postoperati- ve Blutverlust geringer (ca. $250 \mathrm{ml}$; [29, 60, 61]). Mit roboterassistierter Technik operierte Patienten konnten bis zu einem Tag früher aus dem Spital entlassen werden und benötigten weniger Rehabilitationsaufenthalte $[18,27]$. Inwiefern 
hier ein gewisser Placeboeffekt vorliegt, ist $\mathrm{zu}$ diskutieren.

\section{Überlebensraten}

\section{Navigation}

In einer Studie von Robert et al. [55], die auf dem New Zealand Joint Registry (NZJR) basiert, war bei 19,221 TEP mit einer mittleren Nachbeobachtungszeit von 4,5 bzw. 12 Jahren die Gesamtrevisionsrate wie auch jene bei Patienten unter 65 Jahren in beiden Gruppen ähnlich $[8,33]$. In einer weiteren populationsbasierten Studie mussten sich unter 65Jährige 1,8\% weniger Revisionen unterziehen [1]. In ähnlicher Weise reduzierte die Navigation gemäß einer prospektiven Studie, die auf australischen Registerdaten von 44,573 TEP basierte, auch noch 9 Jahre postoperativ, die Gesamtrevisionsrate und die Revisionsrate wegen Lockerung bei den unter 65-Jährigen [62]

\section{Robotik}

Kim et al. [34] verzeichneten in einer randomisierten kontrollierten Studie mit 1516 Patienten unter 65 Jahren bei einer Nachbeobachtungszeit von 15 Jahren in beiden Gruppen eine Überlebensrate von $98 \%$.

\section{Computerassistierte Chirurgie vs.} konventionelle Technik in der UKA

\section{Navigation}

Es gibt wenig vergleichende Studien zwischen der nav-UKA und der konv-UKA. Auch hier wird die genauere postoperative Ausrichtung der mechanischen Achse und der Prothesenkomponenten bei der nav-UKA hervorgehoben $[23,31]$ sowie auf eine Reduktion der Anzahl AusreiBer in der mechanischen Achse und in aller Ebenen der Komponentenausrichtung hingewiesen [56]. Dies war auch der Fall in der Studie von Song et al. [59], die 9 Jahre postoperativ eine optimalere koronare, jedoch nicht sagittale Ausrichtung der Komponenten, weniger Ausreißer, bessere klinische Ergebnisse (HSS, WOMAC, Schmerzen) und 10Jahres-Überlebensrate der Prothese beobachtete.

\section{Robotik}

Die meisten Studien zu UKA hatten den Vergleich zwischen Robotik und konventioneller Technik zum Gegenstand. Die Mehrheit der Studien belegte, dass mit der Robotik eine signifikant verbesserte postoperative Ausrichtung des Beins und eine idealere Komponentenpositionierung erreicht wurden und dass weniger Abweichungen der präoperativen Planung auftraten $[3,5,9,11,19,22,31$, $39,48,66]$.

Mit der rob-UKA betrugen die Abweichungen der Beinachse postoperativ $0,2^{\circ}-0,9^{\circ}$, wobei sich über $87 \%$ der Knie innerhalb von $\pm 2^{\circ}$ befanden. In Bezug auf dieses Kriterium lassen sich bei der konvUKA Werte von $2,7-2,8^{\circ}$ und $40-60 \%$ nachweisen [11, 31, 39].

In einer randomisierten kontrollierten Studie von Bell et al. [5] war der Anteil der Patienten mit einer Komponentenpositionierung innerhalb von $2^{\circ}$ der Zielposition in der Gruppe mit robUKA signifikant größer als in der Gruppe mit konv-UKA, und zwar in allen Ebenen der femoralen Komponente und in der sagittalen und axialen Ebene der Tibiakomponente [5].

Bei den rob-UKA wurde eine geringere Resektionsbreite des Tibiaplateaus festgestellt sowie eine bessere Restitution der Höhe der Gelenklinie und des Slopes beobachtet $[19,39,54]$.

\section{》) Die Operationszeit war bei der roboterassistierten UKA im Durchschnitt länger}

Die Operationszeit war auch bei der robUKA im Durchschnitt länger [11, 67, 69], jedoch konnte die Hospitalisationsdauer verkürzt werden [30].

Eine multizentrische Studie wies nach, dass nach 5,7 Jahren die Überlebensrate bei $97 \%$ lag. Patienten, die sich keiner Revision unterziehen mussten, waren zu diesem Zeitpunkt zu 91\% entweder sehr zufrieden oder zufrieden mit ihrer Kniefunktion [35, 53]. Weitere Studien eruierten nach 2 Jahren eine Überlebensrate von über $95 \%$ mit der rob-UKA und zwischen $89 \%$ und 96,3\% mit der konv.UKA $[3,16,45]$. In den funktionellen und patientenbezogenen Ergebnismessungen wurde hinsichtlich WOMAC, OKS und KSS kein signifikanter Unterschied bis 2 Jahren postoperativ beschrieben [11, 16, 52, 67, 69].

Die Robotik bietet dem Chirurgen mit geringeren Fallzahlen die Möglichkeit, diese mangelnde Erfahrung dank der Robotik wettzumachen $[25,26]$. Auch bei ihnen wurde eine präzisere Position der Komponenten und günstige Lernkurve bezüglich der Operationszeit beobachtet $[25,28]$.

Computerassistierte Navigation vs. Robotik in der Knieendoprothetik

Es findet sich nur eine Studie, die Vergleiche zwischen der nav-TEP und der rob-TEP anstellt. Clark et al. [10] fanden heraus, dass die Robotik eine der neutralen mechanischen Achse um $0,5^{\circ}$ nähere postoperative Beinachse erreicht und eine kürzere Operationszeit sowie einen kürzeren Krankenhausaufenthalt (0,6 Tage) erfordert [10].

\section{Diskussion}

Die Navigation erlaubt es dem Chirurgen, synchron eine Rückmeldung zu einzelnen Operationsschritten zu erhalten, so dass er das Operationsergebnis optimieren und intraoperative Fehler verringern kann. Die heutigen Systeme nehmen dem Chirurgen die Planung nicht ab. Bei Zuhilfenahme der Robotik übernimmt jedoch der Roboter zusätzlich gewisse Schritte der Ausführung (Säge oder Fräse) und bietet so zusätzliche Präzision und auch eine gewisse Sicherheit, kein Gewebe zu verletzen.

Es ist allgemein anerkannt, dass die Verwendung der computerassistierten Navigation im Vergleich zur konventionellen Technik sowohl hinsichtlich der TEP wie der UKA eine präzisere und reproduzierbare Wiederherstellung der mechanischen Achse und der Positionierung der Prothesenkomponenten ermöglicht. Mit der Robotik wird theoretisch zusätzlich zur präziseren Achsenausrichtung und Positionierung der Prothesenkomponenten die unabsichtliche Verletzung von Weichteilgewebe vermindert [27, 32]. Dies wird insbesondere durch die Limitation des 
Bewegungswegs der Säge und durch die planbare Prothesenposition erreicht. Chirurgen, die in der unikondylären Prothetik über weniger Erfahrung und Fallzahlen verfügen, könnten von der Robotik profitieren.

Eine fundamentale Frage bleibt offen: Beeinflussen die bessere Ausrichtung der mechanischen Beinachse und der Prothesenkomponenten sowie die Methode die funktionellen und patientenbezogenen Ergebnismessungen oder die Überlebenszeit der Prothese? Aktuell fehlen in dieser Hinsicht schlüssige Nachweise, insbesondere auch hinsichtlich der Robotik, die praktisch nur von kurzfristigen Studien abgedeckt wird. Es muss festgehalten werden, dass sich zum einen etliche Studien nur auf eine kleine Anzahl Patienten abstützen, die zudem bei der Anwendung der Robotik sicher streng selektioniert wurden, und zum anderen die postoperativen Messungen $\mathrm{zu}$ heterogen sind. Zudem sind die Anwender meist erfahrene Kniechirurgen. Es braucht deshalb weitere Studien von besserer Qualität zum Nachweis der Unterschiede zwischen konventioneller und computerassistierter Endoprothetik in Bezug auf die langfristigen funktionellen und patientenbezogenen Ergebnismessungen, die Überlebensdauer der Prothese und die Kosteneffektivität.

Wegen der beachtlichen Anschaffungs- und Unterhaltskosten, insbesondere für die Robotik, der zu erwartenden Weiterentwicklung des Robotersystems, der unbefriedigenden Studienqualität sowie der ausstehenden Langzeitergebnisse, kann man aktuell keine Empfehlung zur flächendeckenden Einführung geben. Auch spielt bei der UKA das Fallvolumen für die Rentabilität eine große Rolle [47]. Ein bildgestütztes Navigationssystem bietet dem Chirurgen schon heute eine gute Unterstützung bei der Planung und Durchführung der einzelnen Operationsschritte.

\section{Fazit für die Praxis}

\section{- Computerassistierte Navigation und Robotik führen zu einer präzise- ren Ausrichtung der mechanischen Beinachse und der Prothesenkompo- nenten mit weniger Ausreißern.}

- Die Robotik schützt zusätzlich vor ungewollten Knochen- und Weichteilverletzungen.

- Die kurz- und mittelfristigen klinischen Ergebnismessungen in der Funktion und die Resultate der PROMs sind kontrovers und zeigen kaum Unterschiede zur konventionellen Technik.

- Es resultiert keine oder nur eine geringe Verlängerung der Überlebensrate der Prothesen.

- Beachtliche Anschaffungs- und Unterhaltskosten, insbesondere bei der Robotik, höhere Strahlenexposition durch die präoperative CTBildgebung, längere Planungszeiten, Verlängerung der intra- und perioperativen Zeitdauer und ein möglicher Ausfall des Systems limitieren die Anwendung der computerassistierten Systeme.

- Eine solide Erfahrung in der konventionellen Endoprothetik ist Bedingung, um Ausführungsfehler zu erkennen und ggf. einzugreifen.

- Es sind weitere Studien zu Langzeitergebnissen und verschiedenen Alignementstrategien erforderlich.

\section{Korrespondenzadresse}

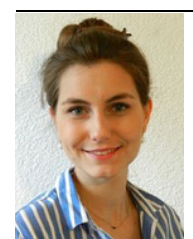

\section{Dr. Céline S. Moret}

Klinik für Orthopädie und Traumatologie, Kantonsspital Baselland

4101 Bruderholz, Schweiz celinesaphena.moret@ unibas.ch

Funding. Open access funding provided by University of Basel

\section{Einhaltung ethischer Richtlinien}

Interessenkonflikt. C.S. Moret und M.T. Hirschmann geben an, dass kein Interessenkonflikt besteht.

Für diesen Beitrag wurden von den Autoren keine Studien an Menschen oder Tieren durchgeführt. Für die aufgeführten Studien gelten die jeweils dort angegebenen ethischen Richtlinien.

Open Access. Dieser Artikel wird unter der Creative Commons Namensnennung 4.0 International Lizenz veröffentlicht, welche die Nutzung, Vervielfältigung, Bearbeitung, Verbreitung und Wiedergabe in jeglichem Medium und Format erlaubt, sofern Sie den/die ursprünglichen Autor(en) und die Quelle ordnungsgemäßnennen, einen Link zur Creative Commons Lizenz beifügen und angeben, ob Änderungen vorgenommen wurden.

Die in diesem Artikel enthaltenen Bilder und sonstiges Drittmaterial unterliegen ebenfalls der genannten Creative Commons Lizenz, sofern sich aus der Abbildungslegende nichts anderes ergibt. Sofern das betreffende Material nicht unter der genannten Creative Commons Lizenz steht und die betreffende Handlung nicht nach gesetzlichen Vorschriften erlaubt ist, ist für die oben aufgeführten Weiterverwendungen des Materials die Einwilligung des jeweiligen Rechteinhabers einzuholen.

Weitere Details zur Lizenz entnehmen Sie bitte der Lizenzinformation auf http://creativecommons.org/ licenses/by/4.0/deed.de.

\section{Literatur}

1. Antonios JK, Kang HP, Robertson D, Oakes DA, Lieberman JR, Heckmann ND (2020) Populationbased survivorship of computer-navigated versus conventional total knee arthroplasty. J Am Acad Orthop Surg 28:857-864

2. Baker PN, van der Meulen JH, Lewsey J, Gregg PJ, National Joint Registry for England and Wales (2007) The role of pain and function in determining patient satisfaction after total knee replacement. Data from the national joint registry for England and Wales. JBone Joint Surg Br 89:893-900

3. Batailler C, White N, Ranaldi FM, Neyret P, Servien E, Lustig S (2019) Improved implant position and lower revision rate with robotic-assisted unicompartmental knee arthroplasty. Knee Surg Sports Traumatol Arthrosc 27:1232-1240

4. Bauwens K, Matthes G, Wich M, Gebhard F, Hanson B, Ekkernkamp A, Stengel D (2007) Navigated total knee replacement. A metaanalysis. J Bone Joint Surg Am 89:261-269

5. Bell SW, Anthony I, Jones B, MacLean A, Rowe $P$, Blyth M (2016) Improved accuracy of component positioning with robotic-assisted unicompartmental knee arthroplasty: data from a prospective, randomized controlled study. J Bone Joint Surg Am 98:627-635

6. Berend ME, Ritter MA, Meding JB, Faris PM, Keating EM, Redelman R, Faris GW, Davis KE (2004) Tibial component failure mechanisms in total knee arthroplasty. Clin Orthop Relat Res 428:26-34

7. Bourne RB, Chesworth BM, Davis AM, Mahomed NN, Charron KDJ (2010) Patient satisfaction after total knee arthroplasty: who is satisfied and who is not? Clin Orthop Relat Res 468:57-63

8. Cip J, Obwegeser F, Benesch T, Bach C, Ruckenstuhl P, Martin A (2018) Twelve-year follow-up of navigated computer-assisted versus conventional total knee arthroplasty: a prospective randomized comparative trial. J Arthroplasty 33:1404-1411

9. Citak M, Suero EM, Citak M, Dunbar NJ, Branch SH, Conditt MA, Banks SA, Pearle AD (2013) Unicompartmental knee arthroplasty: is robotic technology more accurate than conventional technique? Knee 20:268-271

10. Clark TC, Schmidt FH (2013) Robot-assisted navigation versus computer-assisted navigation in primary total knee arthroplasty: efficiency and accuracy. ISRN Orthop 2013:794827

11. Cobb J, Henckel J, Gomes P, Harris S, Jakopec M, Rodriguez F, Barrett A, Davies B (2006) Handson robotic unicompartmental knee replacement. JBone Joint Surg Br 88:188-197 
12. Confalonieri $N$, Chemello $C$, Cerveri $P$, Manzotti A (2012) Is computer-assisted total knee replacement for beginners or experts? Prospective study among three groups of patients treated by surgeons with different levels of experience. J Orthop Traumatol 13:203-210

13. Dy CJ, Marx RG, Bozic KJ, Pan TJ, Padgett DE, Lyman S (2014) Risk factors for revision within 10 years of total knee arthroplasty. Clin Orthop Relat Res 472:1198-1207

14. Ehrendorfer $S$, Erdmenger $U$, Bocanegra LM, Boyd M, Canal J, Canal J, Hoch A, Windhager R, Niehaus R (2020) Limb and component alignment after total knee arthroplasty comparing 28 consecutive iassist and 28 conventional TKAs: a prospective study. Knee 27:1881-1888

15. Ethgen $O$, Bruyère $O$, Richy $F$, Dardennes $C$, Reginster J-Y (2004) Health-related quality of life in total hip and total knee arthroplasty. A qualitative and systematic review of the literature. JBone Joint Surg Am 86:963-974

16. Gilmour A, MacLean AD, Rowe PJ, Banger MS, Donnelly I, Jones BG, Blyth MJG (2018) Roboticarm-assisted vs conventional unicompartmental knee arthroplasty. The 2-year clinical outcomes of a randomized controlled trial. J Arthroplasty 33:S109-S115

17. Grimberg A, Jansson V, Lützner J, Melsheimer O, Morlock M, Steinbrück A (2020) Endoprothesenregister Deutschland - Jahresbericht 2020. EPRD $\mathrm{gGmbH}$,

18. Grosso MJ, Li WT, Hozack WJ, Sherman M, Parvizi J, Courtney PM (2020) Short-term outcomes are comparable between robotic-arm assisted and traditional total knee arthroplasty. J Knee Surg. https://doi.org/10.1055/s-0040-1718603

19. Herry $Y$, Batailler $C$, Lording T, Servien $E$, Neyret $P$, Lustig S (2017) Improved joint-line restitution in unicompartmental knee arthroplasty using a robotic-assisted surgical technique. Int Orthop 41:2265-2271

20. Hofmann S, Seitlinger G, Djahani O, Pietsch M (2011) The painful knee after TKA: a diagnostic algorithm for failure analysis. Knee Surg Sports Traumatol Arthrosc 19:1442-1452

21. Hsu RW-W, Hsu W-H, Shen W-J, Hsu W-B, Chang S-H (2019) Comparison of computer-assisted navigation and conventional instrumentation for bilateral total knee arthroplasty: the outcomes at mid-term follow-up. Medicine 98:e18083

22. Iñiguez M, Negrín R, Duboy J, Reyes NO, Díaz R (2019) Robot-assisted unicompartmental knee arthroplasty: increasing surgical accuracy? A cadaveric study. J Knee Surg. https://doi.org/10 1055/s-0039-1698771

23. Jung KA, Kim SJ, Lee SC, Hwang SH, Ahn NK (2010) Accuracy of implantation during computerassisted minimally invasive Oxford unicompartmental knee arthroplasty: a comparison with a conventional instrumented technique. Knee 17:387-391

24. Kahlenberg CA, Nwachukwu BU, McLawhorn AS, Cross MB, Cornell CN, Padgett DE (2018) Patient satisfaction after total knee replacement: a systematic review. HSS J 14:192-201

25. Karia M, Masjedi M, Andrews B, Jaffry Z, Cobb J (2013) Robotic assistance enables inexperienced surgeons to perform unicompartmental knee arthroplasties on dry bone models with accuracy superior to conventional methods. Adv Orthop 2013:481039

26. Kayani B, Haddad FS (2019) Robotic unicompartmental knee arthroplasty: current challenges and future perspectives. Bone Joint Res 8:228-231
27. Kayani B, Konan S, Pietrzak JRT, Haddad FS (2018) latrogenic bone and soft tissue trauma in robotic-arm assisted total knee arthroplasty compared with conventional jig-based total knee arthroplasty: a prospective cohort study and validation of a new classification system. J Arthroplasty 33:2496-2501

28. Kayani B, Konan S, Pietrzak JRT, Huq SS, Tahmassebi J, Haddad FS (2018) The learning curve associated with robotic-arm assisted unicompartmental knee arthroplasty: a prospective cohort study. Bone Joint J 100-B:1033-1042

29. Kayani B, Konan S, Tahmassebi J, Pietrzak JRT, Haddad FS (2018) Robotic-arm assisted total knee arthroplasty is associated with improved early functional recovery and reduced time to hospital discharge compared with conventional jig-based total knee arthroplasty: a prospective cohort study. Bone Joint J 100-B:930-937

30. Kayani B, Konan S, Tahmassebi J, Rowan FE, Haddad FS (2019) An assessment of early functional rehabilitation and hospital discharge in conventional versus robotic-arm assisted unicompartmental knee arthroplasty: a prospective cohort study. Bone Joint J 101-B:24-33

31. Keene G, Simpson D, Kalairajah Y (2006) Limb alignment in computer-assisted minimallyinvasive unicompartmental knee replacement. JBone Joint Surg Br 88-B:44-48

32. Khlopas A, Chughtai M, Hampp EL, Scholl LY, Prieto M, Chang T-C, Abbasi A, Bhowmik-Stoker M, Otto J, Jacofsky DJ, Mont MA (2017) Robotic-arm assisted total knee arthroplasty demonstrated soft tissue protection. Surg Technol Int 30:441-446

33. Kim Y-H, Park J-W, Kim J-S (2012) Computer-navigated versus conventional total knee arthroplasty a prospective randomized trial. J Bone Joint Surg Am 94:2017-2024

34. Kim Y-H, Yoon S-H, Park J-W (2020) Does roboticassisted TKA result in better outcome scores or long-term survivorship than conventional TKA? A randomized, controlled trial. Clin Orthop Relat Res 478:266-275

35. Kleeblad LJ, Borus TA, Coon TM, Dounchis J, Nguyen JT, Pearle AD (2018) Midterm survivorship and patient satisfaction of robotic-arm-assisted medial unicompartmental knee arthroplasty: a multicenter study. J Arthroplasty 33:1719-1726

36. Krackow KA, Bayers-Thering M, Phillips MJ, BayersThering M, Mihalko WM (1999) A new technique for determining proper mechanical axis alignment during total knee arthroplasty: progress toward computer-assisted TKA. Orthopedics 22:698-702

37. Lee B-S, Cho H-I, Bin S-I, Kim J-M, Jo B-K (2018) Femoral component varus malposition is associated with tibial aseptic loosening after TKA Clin Orthop Relat Res 476:400-407

38. Lee D-Y, Park Y-J, Hwang S-C, Park J-S, Kang D-G (2020) No differences in mid- to long-term outcomes of computer-assisted navigation versus conventional total knee arthroplasty. Knee Surg Sports Traumatol Arthrosc 28:3183-3192

39. Lonner JH, John TK, Conditt MA (2010) Robotic arm-assisted UKA improves tibial component alignment: a pilot study. Clin Orthop Relat Res 468:141-146

40. Lüring C, Bäthis H, Tingart M, Prelick L, Grifka J (2005) Die navigationsgestützte Knieendoprothetik: Eine Standortbestimmung unter evidenzbasierten Kriterien. Dtsch Arztebl 102:34-35

41. Marchand RC, Sodhi N, Khlopas A, Sultan AA, Higuera CA, Stearns KL, Mont MA (2018) Coronal correction for severe deformity using roboticassisted total knee arthroplasty. J Knee Surg 31:2-5
42. Mason JB, Fehring TK, Estok R, Banel D, Fahrbach K (2007) Meta-analysis of alignment outcomes in computer-assisted total knee arthroplasty surgery. JArthroplasty 22:1097-1106

43. Matziolis G, Krocker D, Weiss U, Tohtz S, Perka C (2007) A prospective, randomized study of computer-assisted and conventional total knee arthroplasty. Three-dimensional evaluation of implant alignment and rotation. J Bone Joint Surg Am 89:236-243

44. McClelland JA, Webster KE, Ramteke AA, Feller JA (2017) Total knee arthroplasty with computerassisted navigation more closely replicates normal knee biomechanics than conventional surgery. Knee 24:651-656

45. Mergenthaler G, Batailler C, Lording T, Servien E, Lustig $S$ (2021) Is robotic-assisted unicompartmental knee arthroplasty a safe procedure? A case control study. Knee Surg Sports Traumatol Arthrosc 29:931-938

46. Meyer M, Renkawitz T, Völlner F, Benditz A, Grifka J, Weber M (2021) Pros and cons of navigated versus conventional total knee arthroplasty-a retrospective analysis of over 2400 patients. Arch Orthop Trauma Surg. https://doi.org/10.1007/ s00402-021-03834-y

47. MoschettiWE, Konopka JF, Rubash HE, Genuario JW (2016) Can robot-assisted unicompartmental knee arthroplasty be cost-effective? A Markov decision analysis. J Arthroplasty 31:759-765

48. Negrín R, Duboy J, Iñiguez $M$, Reyes NO, Barahona M, Ferrer G, Infante C, Jabes N (2021) Robotic-assisted vs conventional surgery in medial unicompartmental knee arthroplasty: a clinical and radiological study. Knee Surg Relat Res 33:5

49. Novoa-Parra CD, Sanjuan-Cerveró R, FrancoFerrando N, Larrainzar-Garijo R, Egea-Castro G, Lizaur-Utrilla A (2020) Complications of computerassisted navigation in total knee replacement: retrospective cohort of eight hundred and seventy eight consecutive knees. Int Orthop 44:2621-2626

50. Pabinger C, Berghold A, Boehler N, Labek G (2013) Revision rates after knee replacement. Cumulative results from worldwide clinical studies versus joint registers. Osteoarthritis Cartilage 21:263-268

51. Panjwani TR, Mullaji A, Doshi K, Thakur H (2019) Comparison of functional outcomes of computerassisted vs conventional total knee arthroplasty: a systematic review and meta-analysis of highquality, prospective studies. J Arthroplasty 34:586-593

52. Park KK, Han CD, Yang I-H, Lee W-S, Han JH, Kwon HM (2019) Robot-assisted unicompartmental knee arthroplasty can reduce radiologic outliers compared to conventional techniques. PLoS ONE 14:e225941

53. Pearle $A D$, van der List JP, Lee $L$, Coon TM, Borus TA, Roche MW (2017) Survivorship and patient satisfaction of robotic-assisted medial unicompartmental knee arthroplasty at a minimum twoyearfollow-up. Knee 24:419-428

54. Ponzio DY, Lonner JH (2016) Robotic technology produces more conservative tibial resection than conventional techniques in UKA. Am J Orthop (Belle Mead NJ) 45:E465-E468

55. Roberts TD, Frampton CM, Young SW (2020) Outcomes of computer-assisted surgery compared with conventional instrumentation in 19,221 tota knee arthroplasties: results after a mean of 4.5 years of follow-up. JBone Joint Surg Am 102:550-556

56. Rosenberger RE, Fink C, Quirbach S, Attal R, Tecklenburg K, Hoser C (2008) The immediate effect of navigation on implant accuracy in primary mini-invasive unicompartmental knee 
57. Scott CEH, Oliver WM, MacDonald D, Wade FA Moran M, Breusch SJ (2016) Predicting dissatisfaction following total knee arthroplasty in patients under 55 years of age. J Bone Joint Surg Br 98B:1625-1634

58. Siston RA, Giori NJ, Goodman SB, Delp SL (2007) Surgical navigation for total knee arthroplasty: a perspective. J Biomech 40:728-735

59. Song EK, Mohite N, Lee S-H, Na B-R, Seon J-K (2016) Comparison of outcome and survival after unicompartmental knee arthroplasty between navigation and conventional techniques with an average 9-year follow-up. J Arthroplasty 31:395-400

60. Song E-K, Seon J-K, Park S-J, Jung WB, Park H-W, Lee GW (2011) Simultaneous bilateral total knee arthroplasty with robotic and conventional techniques: a prospective, randomized study. Knee Surg Sports Traumatol Arthrosc 19:1069-1076

61. Song E-K, Seon J-K, Yim J-H, Netravali NA, Bargar WL (2013) Robotic-assisted TKA reduces postoperative alignment outliers and improves gap balance compared to conventional TKA. Clin Orthop Relat Res 471:118-126

62. de Steiger RN, Liu Y-L, Graves SE (2015) Computer navigation for total knee arthroplasty reduces revision rate for patients less than sixty-five years of age. J Bone Joint Surg Am 97:635-642

63. Stübig T, Windhagen H, KrettekC, Ettinger M (2020) Computerassistierte Chirurgie in der Orthopädie und Traumatologie. Dtsch Arztebl 117:793-800

64. Stulberg SD, Loan P, Sarin V (2002) Computerassisted navigation in total knee replacement: results of an initial experience in thirty-five patients. J Bone Joint Surg Am 84(2):90-98

65. Tandogan RN, Kort NP, Ercin E, van Rooij F, Nover L, Saffarini M, Hirschmann MT, Becker R, Dejour D, European Knee Associates (EKA) (2021) Computer-assisted surgery and patient-specific instrumentation improve the accuracy of tibial baseplate rotation in total knee arthroplasty compared to conventional instrumentation: a systematic review and meta-analysis. Knee Surg Sports Traumatol Arthrosc. https://doi.org/10. 1007/s00167-021-06495-x

66. Thilak J, Thadi M, Mane PP, Sharma A, Mohan V, Babu BC (2020) Accuracy of tibial component positioning in the robotic arm assisted versus conventional unicompartmental knee arthroplasty. J Orthop 22:367-371

67. Wong J, Murtaugh T, Lakra A, Cooper HJ, Shah RP, Geller JA (2019) Robotic-assisted unicompartmental knee replacement offers no early advantage over conventional unicompartmental knee replacement. Knee Surg Sports Traumatol Arthrosc 27:2303-2308

68. Yang HY, Seon JK, Shin YJ, Lim HA, Song EK (2017) Robotic total knee arthroplasty with a cruciateretaining implant: a 10-year follow-up study. Clin Orthop Surg 9:169-176

69. Zhang F, Li H, Ba Z, Bo C, Li K (2019) Robotic armassisted vs conventional unicompartmental knee arthroplasty: a meta-analysis of the effects on clinical outcomes. Medicine 98:e16968

Hier steht eine Anzeige. Springer 